\title{
Dose Response Study of Intrathecal Morphine for Off-Pump Coronary Artery Bypass Grafting: a pilot Prospective Randomized Double-Blinded Controlled Clinical Trial
}

\author{
William $\mathrm{Ng}^{1}$, Jo Carroll $\mathrm{RN}^{2}$ and George Djaiani ${ }^{2 *}$ \\ 1Department of Anesthesia, Congenital Cardiac Division, University of California San Francisco, USA \\ 2Department of Anesthesia and Pain Management, Toronto General Hospital, University of Toronto, Canada
}

Submission: November 19, 2016; Published: February 22, 2017

*Corresponding author: George Djaiani, Department of Anesthesia and Pain Management, University of Toronto, University Health Network, 200 Elizabeth Street, EN3-438, Toronto, ON M5G 2C4, Canada, Fax: +1 (416) 340 3698; Tel: +1 (416) 340 3567; Email: George.Djaiani@uhn.ca

Abstract

Objective: To determine the optimal dose of intrathecal morphine in patients undergoing off-pump coronary artery bypass grafting (OPCAB) surgery.

Design: A prospective, randomized, double-blinded, placebo-controlled study.

Setting: A tertiary-level university teaching hospital.

Participants: 31 adult patients scheduled for elective primary OPCAB surgery.

Interventions: Patients were randomized to receive $2.5 \mu \mathrm{g} / \mathrm{kg}$ (ITM-2.5) or $5 \mu \mathrm{g} / \mathrm{kg}$ (ITM-5) intrathecal morphine or sham-control preoperatively. Anaesthesia was induced and maintained with sufentanil (limited to $1 \mu \mathrm{g} / \mathrm{kg}$ ), propofol, rocuronium and isoflurane. Blinded observer-caregivers extubated the patients using protocol-defined criteria, and on-demand intravenous morphine (IVM) was administered to patients using a standardized protocol.

Measurements and Results: Blinded observers measured the time to extubation, the amount of supplemental IVM used in the first 48hours post-extubation, satisfaction with pain control, ICU discharge time, hospital discharge time, the incidence of vomiting, pruritus, urinary retention. Pain at rest (VASR) and during movement (VASM) were scored using the Visual Analogue Scale at 2, 4, 8, 24, 36 and 48 hours from extubation. ITM $2.5 \mu \mathrm{g} / \mathrm{kg}$ was as effective as $5 \mu \mathrm{g} / \mathrm{kg}$, and superior to on demand IVM alone. VAS values in ITM-2.5 and ITM-5 groups were similar, and significantly lower in first 24hours comparing to the control group at rest as well as during coughing ( $<<0.05)$. The 48hour cumulative dose of IVM was $22 \pm 12 \mathrm{mg}, 28 \pm 14 \mathrm{mg}$, and $36 \pm 18 \mathrm{mg}$ in ITM-5, ITM-2.5, and control groups respectively. Fewer cases of pruritus, PONV and urinary retention were reported in the ITM-2.5 compared with the ITM-5 group. There were no differences among the study groups with regard to extubation time, ICU discharge and hospital discharge time.

Conclusion: ITM of $2.5 \mu \mathrm{g} / \mathrm{kg}$ was superior to IVM analgesia regimen. Furthermore, it had similar effectiveness and fewer side effects compared to ITM of $5 \mu \mathrm{g} / \mathrm{kg}$. This study suggests that the optimal effective dose of ITM is $2.5 \mu \mathrm{g} / \mathrm{kg}$ for OPCAB surgery for postoperative analgesia. Keywords: Intrathecal morphine; Neuraxial opioid; Coronary artery bypass graft; Off-pump surgery; Postoperative analgesia

Abbrevations: ITM: Intrathecal Morphine; IVM: Intravenous Morphine; OPCAB: Off-Pump Coronary Arterial Bypass; VASR: Visual Analogue Scale at Rest; VASM: Visual Analogue Scale on Movement

\section{Introduction}

Patients undergoing off-pump coronary artery bypass grafting (OPCAB) surgery are suitable for Ultra-Fast-Track protocol including tracheal extubation in the operating room

$[1,2]$. This approach has been associated with a restriction of intraoperative opioid administration, in order to preserve the respiratory drive upon awakening after surgery and may lead to 
inadequate postoperative analgesia. Intrathecal Morphine (ITM) provides effective postoperative analgesia in major surgery $[3,4]$ with a low risk of epidural haematoma $[5,6]$. Recent studies have shown that ITM provides effective postoperative analgesia in cardiac surgery $[7,8]$. But what is unclear from the literature is the minimal effective dose of ITM for cardiac surgery, especially in the context of OPCAB surgery, whose patients are suited to a fast-track extubation protocol.

Our goal was to determine the optimal dose, whilst minimizing the side effects of ITM in patients undergoing OPCAB surgery. We hypothesize that $2.5 \mu \mathrm{g} / \mathrm{kg}$ of ITM would not be inferior to $5 \mu \mathrm{g} / \mathrm{kg}$ of ITM with respect to adequacy of pain control and side-effect profile.

\section{Methods}

After receiving permission from the UHN Research Ethics Board, we recruited 31 patients scheduled for OPCAB surgery and obtained their written consent to participate in this prospective randomized double-blinded controlled clinical trial.

Patients were excluded from the study if they had contraindications to regional anaesthesia such as local sepsis, anticoagulation, non-elective surgery, left ventricular ejection fraction (LVEF) less than $40 \%$ or if they refused consent.

A total of 31 patients were studied (Table 1). Of the 31 patients, 11 were randomized to the (ITM-5) $5 \mu \mathrm{g} / \mathrm{kg}$ intrathecal morphine group, 10 to the (ITM-2.5) $2.5 \mu \mathrm{g} / \mathrm{kg}$ and 10 to the sham-control groups respectively. A computer-generated randomization was used in the allocation to the groups. All patients, intraoperative caregivers and ICU caregivers were unaware of the group allocations. All groups had comparable demographic data, co-morbidities and surgical characteristics (Table 1).

Table 1: Patient and Surgery Characteristics.

\begin{tabular}{|c|c|c|c|}
\hline Type of Analgesia & ITM $5 \mu \mathrm{g} / \mathrm{kg}$ & ITM $2.5 \mu \mathrm{g} / \mathrm{kg}$ & Control \\
\hline Number of Patients & 11 & 10 & 10 \\
\hline Age (years) & $63.1 \pm 11.7$ & $64.4 \pm 9.3$ & $61.8 \pm 8.9$ \\
\hline Gender: Male (\%) & $8(73)$ & $8(80)$ & $9(90)$ \\
\hline Weight (kg) & $78.4 \pm 10.8$ & $78.1 \pm 11.7$ & $83.8 \pm 10.4$ \\
\hline Height $(\mathrm{cm})$ & 164 & 169 & 172 \\
\hline $\mathrm{BSA}(\mathrm{m} 2)$ & $1.87 \pm 0.7$ & $1.89 \pm 0.4$ & $1.93 \pm 0.5$ \\
\hline LV Grade 1 (\%) & $6(55)$ & $5(50)$ & $5(50)$ \\
\hline LV Grade 2 (\%) & $5(45)$ & $5(50)$ & $5(50)$ \\
\hline $\begin{array}{l}\text { Duration of surgery } \\
\qquad(\mathrm{min})\end{array}$ & $221 \pm 34$ & $239 \pm 51$ & $251 \pm 59$ \\
\hline $\begin{array}{l}\text { Number of grafts: } \\
\text { median (range) }\end{array}$ & $3(2 ; 4)$ & $3(2 ; 3)$ & $3(2 ; 3)$ \\
\hline
\end{tabular}

Values are mean \pm SD, where applicable. Percentages and ranges are in brackets.

The preparation of intrathecal morphine was provided by pharmacy staff to ensure blinding. The administrations of ITM were performed by an anesthesiologist not directly designated to the patient's perioperative care. Patients were either placed in the sitting or lateral decubitus positions. The skin was prepared with chlorhexidine, and $2-5 \mathrm{~mL}$ of $2 \%$ lidocaine local anesthetic was injected subcutaneously at L2-3 or L3-4 levels by palpation. A midline approach was used for ITM administration. The designated dose of intrathecal morphine ( 5 or $2.5 \mu \mathrm{g} / \mathrm{kg}$ ) madeup to volumes of $3 \mathrm{~mL}$, this was then administered via a $25 \mathrm{G}$ Whitacre spinal needle via introducer into the intrathecal space. All patients were given local anesthetic subcutaneously, only the sham-control group did not receive an intrathecal puncture. The puncture site was covered by adhesive dressing.

General anesthesia was established with intravenous sufentanil at 0.5 to $1 \mu \mathrm{g} / \mathrm{kg}$, propofol and rocuronium as deemed appropriate by the designated anesthesiologist, and general anesthesia was maintained with isoflurane in oxygen and air mixture. Isoflurane was stopped at time or just prior to skin closure. Propofol infusion was started at $50 \mu \mathrm{g} / \mathrm{kg} / \mathrm{min}$ after skin closure for the transfer to the ICU. There was no restriction on the use of intravenous fluids, blood products, vasopressors, inotropes, vasodilators or anti-arrhythmics in order to maintain haemodynamic stability at the discretion of the designated anesthesiologist.

Sedation was maintained with propofol infusion titrated to a Ramsay sedation score of 3 to 4 . The criteria used for tracheal extubation included:

i. Patient responsive and cooperative.

ii. Negative inspiratory pressures $<-20 \mathrm{~cm} \mathrm{H} 2 \mathrm{O}$.

iii. Vital capacity $>10 \mathrm{~mL} . \mathrm{kg}-1$.

iv. Arterial oxygen tension $(\mathrm{PaO} 2)>80 \mathrm{~mm} \mathrm{Hg}$ and $\mathrm{FiO} 2$ $\leq 0.5, \mathrm{v}$ ) cardiac index $>2.0$ L.min-1.m2.

v. Core temperature $>36.5^{\circ} \mathrm{C}$.

vi. $\mathrm{pH}>7.30$.

vii. Chest drain output $<100 \mathrm{~mL} / \mathrm{h}$ for 2 or more hours.

The ICU nurses performed extubation, and "on-demand" IV morphine (IVM) analgesia according to the standardized protocol [9]. Postoperative pain was treated with morphine, 0.5 to $5 \mathrm{mg}$ intravenously, as required. Shivering was controlled by 25 to $50 \mathrm{mg}$ of intravenous meperidine. Persistent systemic hypertension (systolic blood pressure $>140 \mathrm{~mm} \mathrm{Hg}$ ) was treated with infusion of nitroglycerin and/or nitroprusside, adjusted to a systolic arterial pressure between 90 and $130 \mathrm{~mm} \mathrm{Hg}$. Intravenous boluses of $10-20 \mathrm{mg}$ esmolol were used to control persistent tachycardia $>110$ BPM.

All measurements were performed by an observer who was blinded to the patient's designated group. The analysis of the outcome variables using the intention-to-treat principle. VisualAnalogue-Scale was used to assess analgesia at rest (VASR), and during "on-demand" movement by coughing (VASM) at 2, $4,8,24,36$ and 48 hours from end of extubation. All patients were asked to indicate their pain score on the VAS from the 2 hour mark onwards. At each juncture, pain scores were firstly 
recorded at rest, and immediately followed by a score at cough on-demand. Total supplemental intravenous morphine used and the incidence of morphine related side-effects of vomiting, pruritus and urinary retention within the first 48 hours were recorded. At a day 5 questionnaire, each patient was asked whether they were "satisfied" or "not satisfied" with the analgesia they received.

ICU discharge criteria included:

a. Patient alert and cooperative.

b. No inotropic support and no significant arrhythmia.

c. Adequate ventilation $(\mathrm{PaO} 2>80 \mathrm{~mm} \mathrm{Hg}, \mathrm{PaCO} 2<60 \mathrm{~mm}$ Hg.

d. Chest drain output $<50 \mathrm{~mL} / \mathrm{h}$ for 2 or more hours.

e. Urine output $>0.5 \mathrm{~mL} . \mathrm{kg}-1 . \mathrm{h}-1$.

f. Absence of seizure activity.

The time of ICU discharge was the time of the written order for transfer out of the ICU, as documented by the blinded fellow or anaesthesiologist in-charge. The actual physical discharge time of the patient from the ICU was also recorded. Hospital discharge criteria included:

i. Haemodynamic stability.

ii. Stability of cardiac rhythm.

iii. Absence of wound infection or signs of sepsis.

iv. Adequate urination and defaecation.

v. Independent ambulation and feeding.

Time of hospital discharge was likewise recorded as per the time of written documentation of discharge order by the blinded ward physician in-charge.

The sample size was limited to a pilot study. Categorical and numerical data were analyzed with Fisher's exact test and analysis of variance respectively. A p value of 0.05 was considered statistically significant. Bonferroni correction was applied for multiple testing. Data are expressed as mean \pm SD or median (range).

Results

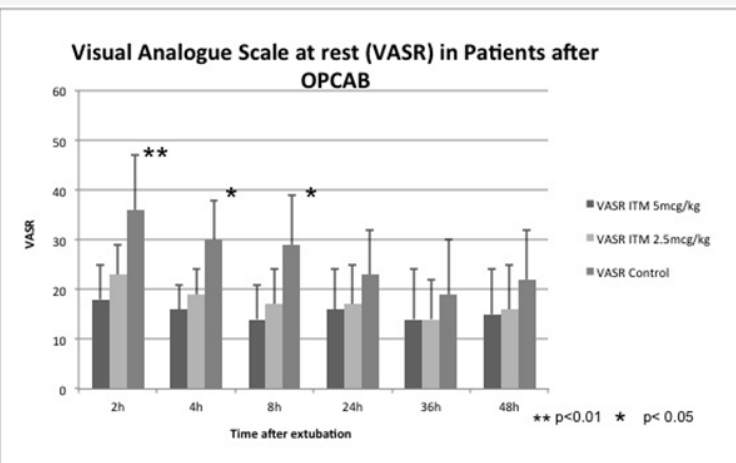

Figure 1: Pain Scores by Visual Analogue Scale at rest (VASR).

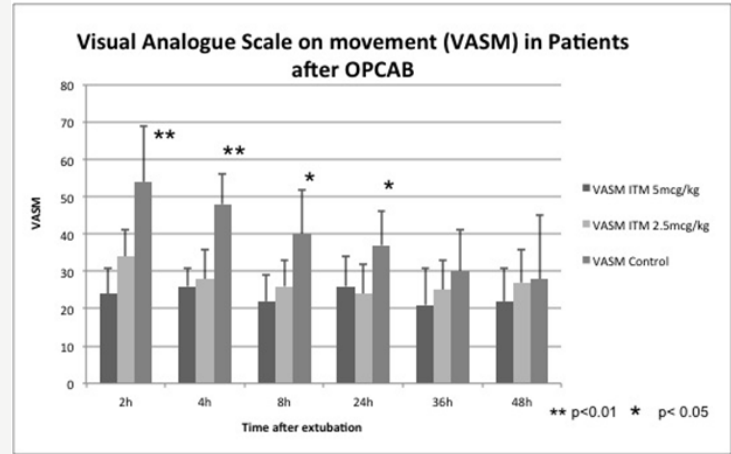

Figure 2: Pain Scores by Visual Analogue Scale on movement (VASM).

Pain scores at rest by VAS (Figure 1) were significantly lower in the ITM-2.5 and ITM-5 groups when compared with control at 2, 4 and 8 hours after extubation. There was a non-significant decreasing trend in pain scores at 24,36 and 48 hours. The ITM2.5 and ITM- 5 groups did not differ significantly in pain scores at rest at all time intervals. P-value was significant at the 0.01 level at 2 hours, and at the 0.05 levels at 4 and 8 hours. Similarly, pain scores on movement by VAS (Figure 2) were significantly lower in the ITM-2.5 and ITM-5 groups when compared with control at $2,4,8$ and 24 hours. P values were significant at the 0.01 level at 2 and 4 hours and at the 0.05 level at 8 and 24 hours. There was a non-significant decreasing trend in pain scores at 36 and 48 hours. Once again the ITM-2.5 and ITM- 5 groups did not differ in pain scores on movement at all time intervals.

The $48 \mathrm{~h}$ cumulative dose of IVM was $22 \pm 12 \mathrm{mg}, 28 \pm 14 \mathrm{mg}$, and $36 \pm 18 \mathrm{mg}$ in ITM-5, ITM-2.5 and control groups respectively (Table 2). However, the decreasing trend in morphine use from ITM-5 to ITM-2.5 to control group did not reach statistical significance.

Table 2: Analysis of Outcomes.

\begin{tabular}{|c|c|c|c|}
\hline Type of Analgesia & $\begin{array}{l}\text { ITM } 5 \mu \mathrm{g} / \\
\quad \mathrm{kg}\end{array}$ & ITM $2.5 \mu \mathrm{g} / \mathrm{kg}$ & Control \\
\hline Number of patients & 11 & 10 & 10 \\
\hline $\begin{array}{l}\text { Total supplemental IV } \\
\text { Morphine } \mathrm{mg} / 48 \mathrm{~h}\end{array}$ & $22 \pm 12$ & $28 \pm 14$ & $36 \pm 18$ \\
\hline Vomiting \# (\%) & $2(18 \%)$ & $1(10 \%)$ & $1(10 \%)$ \\
\hline Pruritus \# (\%) & $3(27 \%)$ & $1(10 \%)$ & $1(10 \%)$ \\
\hline Urinary retention & $2(18 \%)$ & 0 & 0 \\
\hline Extubation time (h) & $4.3 \pm 1.2$ & $3.8 \pm 1.6$ & $3.9 \pm 1.7$ \\
\hline ICU discharge & $1.7 \pm 1.1$ & $1.8 \pm 1.2$ & $1.7 \pm 1.4$ \\
\hline $\begin{array}{l}\text { Hospital discharge } \\
\text { (days) }\end{array}$ & $5.1 \pm 4.1$ & $4.8 \pm 3.9$ & $5.3 \pm 4.2$ \\
\hline Satisfied with analgesia & $9(81 \%)$ & $8(80 \%)$ & $6(60 \%)$ \\
\hline \multicolumn{4}{|c|}{$\begin{array}{c}\text { Values are mean } \pm S D, \text { where applicable. }{ }^{*} \text { Denotes significance with } \\
\text { respect to control. }\end{array}$} \\
\hline
\end{tabular}

With regard to adverse side-effects, 2 of the 11 patients in the ITM-5 group experienced urinary retention compared with 0 of 10 patients respectively in the ITM- 2.5 and control groups. 2 
patients in the ITM-5 groups suffered from PONV compared with 1 patient in the ITM-2.5 and 1 patient in the control group. 3 of the ITM-5 patients complained of pruritus compared with 1 in the ITM-2.5 and 1 in the control group. There was no difference in the extubation time, ICU discharge time, hospital discharge time for the 3 groups in this study.

There were no instances of traumatic tap or spinal haematoma in this study. Activated clotting time were kept at $<400$ seconds by heparin. There were no conversions to onpump CABG.

\section{Discussion}

Intrathecal morphine has been shown to be effective in postoperative analgesia in both cardiac and non-cardiac major surgery. A study comparing two doses of ITM in on-pump CABG surgery suggests $250 \mu \mathrm{g}$ is preferred to $500 \mu \mathrm{g}$ ITM, without delaying tracheal extubation [8]. In a meta-analysis by Liu et al. [10] which included 17 RCTs and a total of 668 patients undergoing, they found that the use of intrathecal morphine in patients undergoing CABG surgery decreased times to extubation, pain scores and IV morphine use postoperatively [10].

However, the optimal dosage of ITM is not known for effective postoperative analgesia, especially in the context of OPCAB surgery. In the previously mentioned meta-analysis10, which varied in opioids used in induction, maintenance and postoperatively, ranges of dosing varied between $250 \mu \mathrm{g}$ and $2000 \mu \mathrm{g}$; weight-adjusted dosing varied $[11,12]$ between $7 \mu \mathrm{g}$. $\mathrm{kg}-1$ to $20 \mu \mathrm{g} . \mathrm{kg}-1$. Given such a wide range of practice in the literature, RCTs such as our present study are called for in the determination of a minimal effective dose of ITM in CABG surgery.

In this study of OPCAB patients, ITM of $2.5 \mu \mathrm{g} / \mathrm{kg}$ was superior to on-demand IVM analgesia regimen for postoperative analgesia and not inferior to ITM of $5 \mu \mathrm{g} / \mathrm{kg}$. VAS values in ITM2.5 and ITM-5 groups were similar, and significantly $(\mathrm{p}<0.05)$ lower in first 24 hours compared to the control group at rest as well as during coughing on-demand (Figures 1 \& 2). However after the 24 hour mark, there was loss of statistical significance despite a continued trend toward lower VASR and VASM in the ITM-2.5 and ITM-5 groups compared to the control group. None of the patients had any apneic episodes related to ITM with the 48 hour observation period. In a similar RCT in patients undergoing OPCAB surgery comparing no ITM with $10 \mu \mathrm{g} / \mathrm{kg}$ ITM led by Turker et al. [13] patients significantly scored less on VASM even at the 24 and 48 hour mark [13]. It appears that if ITM were at a significantly higher level of $10 \mu \mathrm{g} / \mathrm{kg}$ or more, then we expect longer postoperative analgesic effect. The question becomes how to balance optimal postoperative analgesia that provides good conditions for early extubation and patient satisfaction, whilst minimizing the side-effects of ITM.
Crucial to the study findings was blinding of the investigators, the designated case-anesthetist, the operator placing the intrathecal morphine, the patients, the care giver and the observer in ICU. The obvious point of weakness in blinding is the experience of the patient who did not receive intrathecal morphine: he or she would have been given subcutaneous local anaesthetic alone, without the second puncture that necessarily accompanies the delivery of intrathecal morphine. But the subjection of patients to placebo intrathecal puncture was deemed to be an unethical intervention. Hence blinding was considered as best as possible amongst the patient groups, without causing unnecessary harm to the placebo group. Patients would have to have had prior intrathecal puncture experience and recalled the experience exactly, in order to differentiate themselves from the placebo group and the ITM-2.5 or ITM5 groups. From the postoperative questionnaire, there was no suggestion that patient blinding had failed.

In a similar vein, there was a decreasing trend in the cumulative 48 hour intravenous morphine use from control to ITM-2.5 to ITM-5 groups. This was a secondary outcome and surrogate marker of the efficacy of postoperative analgesia of ITM and the trend is in keeping with both older and recent studies of postoperative analgesic efficacy of intrathecal morphine in cardiac surgery $[7,14,15]$ although Chaney [16,17] and Roedinger et al. [18] have revisited their previous conclusions [16-18] on the significance of ITM in post-operative analgesia. Despite not being the primary aim of this study, the findings are in agreement with the general consensus that ITM does provide significant postoperative analgesia in cardiac surgery and is superior to intravenous morphine on-demand alone.

Furthermore the ITM-2.5 group, compared to ITM-5, had lower incidences of PONV and urinary retention, which are wellknown side effects of opioid analgesics. There was a trend toward shorter extubation time in the ITM-2.5 and control group when compared with the ITM-5 group, but this was not statistically significant. The present study findings are in keeping with other studies on ITM in cardiac surgery $[9,19]$ in that ITM did not seem delay extubation; some have gone as far as to say the use of ITM may improve extubation times because of improved pain control in the postoperative period [20]. There is still an ongoing dialogue on whether early extubation is favored or impaired by ITM preoperatively [21]. The sample was underpowered to show the statistical difference between incidence of PONV and pruritus between the ITM-2.5 and ITM-5 groups.

\section{Conclusion}

We support the use of $2.5 \mu \mathrm{g} / \mathrm{kg}$ intrathecal morphine preoperatively as an optimal dose in patients undergoing CABG surgery, especially those suitable for fast-track extubation, such as those undergoing off-pump CABG surgeries. This study was not adequately powered to make definite conclusions regarding major morbidity and mortality. We recommend larger clinical 
trials on the minimal effective dosing of intrathecal morphine in both off-pump and on-pump CABG surgery, to support and verify the finding of this study.

\section{References}

1. Djaiani GN, Ali M, Heinrich L, Bruce J, Carroll J, et al. (2001) Ultra-fasttrack anesthetic technique facilitates operating room extubation in patients undergoing off-pump coronary revascularization surgery. J Cardiothorac Vasc Anesth 15(2): 152-157.

2. Hemmerling TM, Prieto I, Choinière JL, Basile F, Fortier JD (2004) Ultrafast-track anesthesia in off-pump coronary artery bypass grafting: a prospective audit comparing opioid-based anesthesia vs thoracic epidural-based anesthesia. Can J Anaesth 51(2): 163-168.

3. Abboud TK, Dror A, Mosaad P, Zhu J, Mantilla M, et al. (1989) Mini-Dose Intrathecal Morphine for the Relief of Post-Cesarean Section Pain: Safety, Efficacy, and Ventilatory Responses to Carbon Dioxide. Anesth Analg 67(2): 137-143.

4. Kirson LE, Goldman JM, Slover RB (1989) Low-dose intrathecal morphine for postoperative pain control in patients undergoing transurethral resection of the prostate. Anesthesiology 67(2): 192195.

5. Djaiani G, Fedorko L, Beattie WS (2005) Regional anesthesia in cardiac surgery: a friend or a foe? Semin Cardiothorac Vasc Anesth 9(1): 87104.

6. Ho AM, Chung DC, Joynt GM (2000) Neuraxial Blockade and Hematoma in Cardiac Surgery: estimating the risk of a rare adverse event that has not (yet) occurred. Chest 117(2): 551-555.

7. Yapici D, Altunkan ZO, Atici S, Bilgin E, Doruk N, et al. (2008) Postoperative effects of low-dose intrathecal morphine in coronary artery bypass surgery. J Card Surg 23(2): 140-145.

8. Alhashemi JA, Sharpe MD, Harris CL, Sherman V, Boyd D (2000) Effect of subarachnoid morphine administration on extubation time after coronary artery bypass graft surgery. J Cardiothorac Vasc Anesth 14(6): 639-644

9. Cheng DC, Karski J, Peniston C, Asokumar B, Raveendran G, et al. (1996) Morbidity outcome in early versus conventional tracheal extubation after coronary bypass grafting. J Thorac Cardiovasc Surg 112(3): 755764

10. Liu SS, Block BM, Wu CL (2004) Effects of Perioperative Central Neuraxial Analgesia on Outcome after Coronary Artery Bypasss Surgery: a meta-analysis. Anesthesiology 101(1): 153-161.
11. Bowler I, Djaiani G, Abel R, Pugh S, Dunne J, et al. (2002) A Combination of Intrathecal Morphine and Remifentanil Anesthesia for Fast-Track Cardiac Anesthesia and Surgery. J Cardiothorac Vasc Anesth 16(6): 709-714.

12. Casey WF, Wynands JE, Ralley FE, Ramsay JG, O'Connor JP, et al. (1987) The Role of Intrathecal Morphine in the Anesthetic Management of Patients Undergoing Coronary Artery Bypass Surgery. J Cardiothorac Anesth 1(6): 510-516

13. Turker G, Goren S, Sahin S, Korfali G, Sayan E (2005) Combination of intrathecal morphine and remifentanil infusion for fast-track anesthesia in off-pump coronary artery bypass surgery. J Cardiothorac Vasc Anesth 19(6): 708-713.

14. Richardson L, Dunning J, Hunter S (2009) Is intrathecal morphine of benefit to patients undergoing cardiac surgery. Interact Cardiovasc Thorac Surg 8(1): 117-122.

15. Suominen PK, Ragg PG, McKinley DF, Frawley G, But WW, et al. (2004) Intrathecal morphine provides effective and safe analgesia in children after cardiac surgery. Acta Anaesthesiol Scand 48(9): 875-882.

16. Chaney MA, Nikolov MP, Blakeman BP, Bakhos M (1999) Intrathecal morphine for coronary artery bypass graft procedure and early extubation revisited. J Cardiothorac Vasc Anesth 13(5): 574-578.

17. Chaney MA (2005) Cardiac surgery and intrathecal/epidural techniques: at the crossroads? Can J Anaesth 52(8): 783-788.

18. Roediger L, Joris J, Senard M, Larbuisson R, Canivet JL, et al. (2006) The use of pre-operative intrathecal morphine for analgesia following coronary artery bypass surgery. Anaesthesia 61(9): 838-844.

19. Jacobsohn E, Lee TW, Amadeo RJ, Syslak PH, Debrouwere RG, et al. (2005) Low-dose intrathecal morphine does not delay early extubation after cardiac surgery. Can J Anaesth 52(8): 848-857.

20. Parlow JL, Steele RG, O'Reilly D (2005) Low dose intrathecal morphine facilitates early extubation after cardiac surgery: results of a retrospective continuous quality improvement audit. Can J Anaesth 52(1): 94-99.

21. Boulanger A, Perreault S, Choinière M, Priéto I, Lavoie C, et al. (2002) Intrathecal Morphine after Cardiac Surgery. Ann Pharmacother 36(9): 1337-1343.

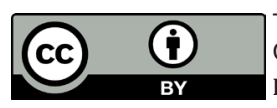

This work is licensed under Creative Commons Attribution 4.0 License DOI: $10.19080 /$ JOCCT.2017.03.555610

\section{Your next submission with Juniper Publishers will reach you the below assets}

- Quality Editorial service

- Swift Peer Review

- Reprints availability

- E-prints Service

- Manuscript Podcast for convenient understanding

- Global attainment for your research

- Manuscript accessibility in different formats ( Pdf, E-pub, Full Text, Audio)

- Unceasing customer service

Track the below URL for one-step submission https://juniperpublishers.com/online-submission.php 